\title{
Zoledronic Acid Suppresses Epithelial-to-Mesenchymal Transition and Invasion via Degradation of Ubiquitinated NEDD9 in PC-3 Prostate Cancer Cells
}

\author{
Tomoaki Tanaka*\#, Kazuya Morimoto\# and Tatsuya Nakatani \\ Department of Urology, Osaka City University Graduate School of Medicine, Osaka, Japan

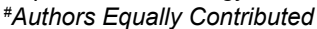

\section{Abstract}

Objective: Zoledronic acid (ZA) is highly effective in the treatment of castration-resistant prostate cancer (CRPC) patients with bone metastases. It is one of bone modifying agents (BMAs) that has been shown to exert not only inhibiting the activation of osteoclasts but also preventing the tumor cell growth, invasion and migration in some cancers. Neural precursor cell-expressed developmentally downregulated protein 9 (NEDD9) is a key regulator of tumor aggressiveness including invasion, epithelial-to-mesenchymal transition (EMT), dedifferentiation and resistance to chemo-drugs. However, research into a biological mechanism in the inhibitory effects of ZA on prostate cancer $(\mathrm{PCa})$ metastasis is still limited. In this study, we examined its effects on tumor cell invasion and EMT via the ubiquitin-proteasomal system for NEDD9 in PC-3 cells.

Methods: We assessed the expression of NEDD9 and its down-stream molecules associated with EMT in PC-3 cells exposure to ZA under the condition with/without TGF- $\beta$. By a boyden chamber assay, the suppressive effect of ZA on PC-3 cell invasion triggered by TGF- $\beta$ was measured. We measured the expression levels of NEDD9 in PC-3 cells exposure to a proteasome inhibitor, MG132. In addition, we detected the effect of ZA on ubiquitinated NEDD9 using an immunoprecipitation method.

Results: ZA markedly inhibited the expression of NEDD9 and its down-stream EMT molecules. Both the invasion and expression of EMT markers of PC-3 cells triggered by TGF- $\beta$ were significantly suppressed by the exposure to ZA. The exposure to MG132 inhibited the degradation of NEDD9 in PC-3 cells. The further add-on of ZA enhanced the polyubiquitination of NEDD9 in PC-3 cells.

Conclusion: The results from a current study indicate that ZA inhibited the invasion and expression of NEDD9 and its EMT markers, along with the enhanced degradation of ubiquitinated NEDD9 in PC-3 cells.

Keywords: Zoledronic acid; EMT; Invasion; NEDD9; Ubiquitination; Proteasome; degradation; PC-3 prostate cancer cells; TGF- $\beta$; BMA

Abbreviations: PCa: Prostate Cancer; ZA: Zoledronic Acid; BMA: Bone Modifying Agent; NEDD9: Neural Precursor Cell-Expressed Developmentally Downregulated Protein 9; EMT: Epithelial-toMesenchymal Transition; CRPC: Castration-Resistant Prostate Cancer; Cas: Crk-Associated Substrate; MET: Mesenchymal-To-Epithelial Transition; UPS: Ubiquitination-Proteasome System; SREs: SkeletalRelated Events; RANK-L:Receptor Activator Of Nuclear Factor-kappa beta-Ligand

\section{Introduction}

Until now, some part of treatment-naïve patients diagnosed with high-risk prostate cancer $(\mathrm{PCa})$ and the most of aggressive castrationresistant prostate cancer (CRPC) patients have bone metastases and their disease state strongly develop the early resistance to hormonal therapy and chemotherapy [1]. From the viewpoint of cell biology, epithelialto-mesenchymal transition (EMT) is an essential mechanism for tumor aggressiveness and metastasis [2]. Aggressive epithelial cancer cells can stimulate embryonic processes, which obtained in epithelial plasticity and change from a polarized, epithelial phenotype to a mesenchymal phenotype with increased motility. Numerous biological alterations, including expression of mesenchymal biomarkers, induction of angiogenesis, increased resistance to apoptosis and marked production of extracellular matrix components, occur in epithelial cancer cells throughout the course of EMT [3,4].

NEDD9 (neural precursor cell-expressed developmentally downregulated protein $\underline{9}$ ) is a core member of the multiple docking protein Cas (C rk-asssociated substrate) family including BCAR1/ p130Cas and CASS4/HEPL, which have been implicated as signaling mediators of diverse biological processes including adhesion, motility, cell cycle, mitosis, apoptosis, anoikis, and tumorigenesis [5-7]. We previously reported that NEDD9 is a crucial player in regulating tumor aggressiveness and invasion, which are triggered by the treatment with TGF- $\beta$, in some prostate cancer cell lines [8].

On the other hand, zoledronic acid (ZA), a major nitrogencontaining bisphosphonate, has been broadly used for the treatment of PCa bone metastases owing to potent inhibition of osteoclast activity and survival $[9,10]$. ZA also has been reported to have the potential of cytotoxic and cytostatic effects on prostate cancer cells [11-13], while those in depth molecular mechanisms remain unclear. In this study, we examined whether ZA can regulate the EMT process and tumor

*Corresponding author: Tomoaki Tanaka, Department of Urology, Osaka City University Graduate School of Medicine, 1-4-3 asahimachi, abeno-ku, Osaka Japan, Tel: +81666453857; E-mail: tomoaki826@msic.med.osaka-cu.ac.jp

Received March 23, 2018; Accepted April 10, 2018; Published April 12, 2018

Citation: Tanaka T, Morimoto K, Nakatani T (2018) Zoledronic Acid Suppresses Epithelial-to-Mesenchymal Transition and Invasion via Degradation of Ubiquitinated NEDD9 in PC-3 Prostate Cancer Cells. J Cancer Sci Ther 10: 080-084. doi: 10.4172/1948-5956.1000523

Copyright: $\odot 2018$ Tanaka T, et al. This is an open-access article distributed under the terms of the Creative Commons Attribution License, which permits unrestricted use, distribution, and reproduction in any medium, provided the original author and source are credited. 
invasion by the control of NEDD9 as a target molecule in prostate cancer cells.

\section{Materials and Methods}

\section{Cell culture and reagents}

A human prostate cancer cell line, PC-3, was obtained from the American Type Culture Collection (ATCC; Manassas, VA, USA). PC-3 cells were maintained in RPMI-1640 medium (Sigma, St. Louis, MO, USA) supplemented with $10 \%$ fetal bovine serum (HyClone, Logan, UT, USA), $100 \mathrm{U} / \mathrm{mL}$ of penicillin and $100 \mu \mathrm{g} / \mathrm{mL}$ of streptomycin (Gibco, New York, NY, USA) at $37^{\circ} \mathrm{C}$ in a humidified atmosphere containing 5\% $\mathrm{CO}_{2}$. TGF- $\beta$ was purchased from Cell Signaling Technology (Beverly, MA, USA) and dissolved in citric acid ( $\mathrm{pH}$ 3.0). The concentration of stock solution was $50 \mu \mathrm{g} / \mathrm{mL}$ and the final concentration was $10 \mathrm{ng} / \mathrm{mL}$ for all the experiments. Zoledronic acid monohydrate was purchased from Sigma-Aldrich (St. Louis, MO, USA) and dissolved in sterile milli-Q water. The concentration of stock solution was $250 \mathrm{mM}$. MG132 was also obtained from Sigma-Aldrich and dissolved in DMSO. The final concentration of stock solution was $2.5 \mathrm{mM}$ and the final concentration was $0.5 \mu \mathrm{M}$ for all the experiments.

\section{Antibodies}

Mouse monoclonal anti-NEDD9(ab18056), anti- $\beta$-actin(ab8226), and rabbit polyclonal anti-Snail+Slug (ab63371) antibodies were obtained from Abcam (Cambridge, UK). Rabbit polyclonal antivimentin (\#5741), anti-E-cadherin (\#3195) antibodies were from Cell Signaling Technology (Beverly, MA, USA). Mouse monoclonal anti-polyubiquitin antibody was purchased from MBL life science (Woburn, MA, USA).

\section{Western blotting}

Cells were harvested and whole-cell lysates were prepared using PRO-PREP protein extraction solution (iNtRON Biotechnology, Gyeonggi-do, Korea) in accordance with the manufacturer's instructions. Protein concentrations of samples were determined by the bicinchoninic acid protein assay (BioRad, Hercules, CA, USA). Protein samples $\left(10 \mu \mathrm{g}\right.$ of each protein) were treated at $55^{\circ} \mathrm{C}$ for 20 min in $2 \%$ SDS-treating solution containing $5 \% \beta$-mercaptoethanol and separated in $10 \%$ SDS- polyacrylamide gels and transferred onto nitrocellulose membranes. Membranes were blocked for $1 \mathrm{~h}$ at room temperature with Tris-buffered saline (TBS) containing $0.05 \%$ Tween 20 and 5\% non-fat dried milk and probed overnight at $4^{\circ} \mathrm{C}$ with primary antibodies under manufacturer-recommended conditions. Immunoblots were washed with TBS containing $0.05 \%$ Tween 20 and $1 \%$ non-fat milk and incubated with secondary antibodies conjugated with horseradish peroxidase against mouse IgG or rabbit IgG (Santa Cruz Biotechnology, Santa Cruz, CA, USA) for $1 \mathrm{~h}$ at room temperature. Immunoreactivity proteins were visualized using the ECL detection system (Pierce, Rockford, IL, USA). Each western blot analysis was performed in triplicate.

\section{Transwell invasion assay}

In vitro cell invasion assay was conducted using Matrigel invasion chambers $(8 \mu \mathrm{M}$ pore size; BD Biosciences Pharmingen, San Diego, CA, USA). Cells were allowed to grow to subconfluency $(\sim 75-80 \%)$ and serum-starved for $24 \mathrm{~h}$. After detachment with trypsin, cells were washed with PBS, resuspended in serum-free medium and $500 \mu \mathrm{l}$ cell suspension $\left(4 \times 10^{4}\right.$ cells $\left./ \mathrm{mL}\right)$ was added to the upper chamber. Complete medium was added to the bottom wells of the chambers. For the screening, after $24 \mathrm{~h}$ the cells that had not infiltrated were removed from the upper face of the filters using cotton swabs, and the cells that had infiltrated were fixed with $5 \%$ glutaraldehyde solution and stained with Giemsa stain solution. Images of four different $\times 100$ fields were captured from each membrane and the number of invading cells was counted.

\section{Immunoprecipitation}

The immunoprecipitation (IP) method was performed in accordance with the manufacture's protocol of ImmunoCruz ${ }^{\mathrm{TM}}$ Immunoprecipitation / Western Blots (Santa Cruz Biotechnology, Santa Cruz, CA, USA). A total cell lysate was prepared in $1 \mathrm{ml}$ of RIPA buffer with a protease inhibitor cocktail (Sigma-Aldrich, St. Louis, MO, USA). Next, to preclean whole cell lysate, $50 \mu$ of the preclean matrix (sc45056) obtained from Santa Cruz Biotechnology was added to $1 \mathrm{ml}$ of whole cell lysate $(25 \% \mathrm{v} / \mathrm{v})$ in a $1.5 \mathrm{~mL}$ microcentrifuge tube, followed by incubation for $30 \mathrm{~min}$ at $4^{\circ} \mathrm{C}$ while rotating. After microcentrifugation at maximum speed for $30 \mathrm{~s}$ at $4^{\circ} \mathrm{C}$, a desired supernatant of IP matrix was transferred to a new microcentrifuge tube. For the formation of IP antibody to IP matrix complex, $50 \mu \mathrm{l}$ of suspended IP matrix, $5 \mu \mathrm{g}$ of anti-NEDD9 antibody described above and $500 \mu \mathrm{l}$ of PBS were added in a microcentrifuge tube. Then, the tube containing a mixture was incubated overnight at $4^{\circ} \mathrm{C}$ on a rotator. After incubation of the anti-NEDD9 antibody with the specific IP matrix, pellet matrix was formed via microcentrifugation at maximum speed for $30 \mathrm{~s}$ at $4^{\circ} \mathrm{C}$. The supernatant was carefully aspirated and discarded. Next, the pelleted matrix was washed 2 times with $500 \mu \mathrm{l}$ of PBS, each time the fore-mentioned centrifugation and aspiration were repeated. After the final wash of the anti-NEDD9 antibody with the specific IP matrix, $1 \mathrm{mg}$ of the total cellular lysate was transferred to the pelleted matrix and incubated overnight at $4^{\circ} \mathrm{C}$ on a rotator. After incubation of the matrix and lysate, the pellet was kept through microcentrifugation at maximum speed for $30 \mathrm{~s}$ at $4^{\circ} \mathrm{C}$. The supernatant was aspirated carefully and discarded. The pellet matrix was washed 4 times with RIPA buffer, each time the same centrifugation and aspiration were repeated. After final wash, the supernatant was carefully aspirated and discarded. The immunoprecipitate was resuspended in $40 \mu \mathrm{l}$ of the sample buffer (sc-24945), followed by the boiling for $3 \mathrm{~min} .5 \mu \mathrm{l}$ of solubilized samples were loaded onto $15 \%$ SDS-polyacrylamide gels. Then, Western blotting was performed in accordance with the manufacture's protocol of ImmunoCruz ${ }^{\mathrm{TM}}$ Immunoprecipitation / Western Blots.

\section{Statistical analysis}

Statistical analysis was performed by Ekuseru-Toukei 2012 (Social Survey Research Information Co., Ltd., Tokyo, Japan). Data were expressed as mean $\pm \mathrm{S}$. D. Student's $t$-test was used to calculate the statistical significance of the experimental results of invasion assays.

\section{Results}

\section{ZA suppresses the expressions of NEDD9 and EMT-associated biomarkers in PC-3 prostate cancer cells}

As shown in Figure 1, the expression levels of NEDD9 and mesenchymal markers such as vimentin and Snail-Slug were downregulated by the exposure to ZA in a dose-dependent manner in PC-3 prostate cancer cells. On the contrary, the expression of E-cadherin, an epithelial marker, markedly increased in the same condition. The changes in expressions of these proteins were obviously detected under the condition with more than $50 \mu \mathrm{M} \mathrm{ZA}$. However, the cytotoxicity of $\mathrm{ZA}$ was severe under the exposure to $100 \mu \mathrm{M} \mathrm{ZA}$ as shown in the lower 
Citation: Tanaka T, Morimoto K, Nakatani T (2018) Zoledronic Acid Suppresses Epithelial-to-Mesenchymal Transition and Invasion via Degradation of Ubiquitinated NEDD9 in PC-3 Prostate Cancer Cells. J Cancer Sci Ther 10: 080-084. doi: 10.4172/1948-5956.1000523

panel of Figure 1 (i.e., small amount of $\beta$ actin). Thus, we selected the condition of $50 \mu \mathrm{M} \mathrm{ZA}$ in the followed experiments.

\section{ZA strongly inhibits the TGF- $\beta$-triggered EMT process in PC-3 cells}

In PC-3 prostate cancer cells, the addition with $\mathrm{ZA}(50 \mu \mathrm{M})$ clearly reversed the TGF- $\beta$-induced EMT (namely mesenchymal-toepithelial transition; MET), concomitant with the suppression of both the increase in the expression of vimentin and the decrease in that of E-cadherin, respectively (Figure 2A).

\section{ZA markedly suppresses the TGF- $\beta$-induced tumor-cell invasion}

Under the same condition as shown in Figure $2 \mathrm{~B}$, the treatment with ZA significantly inhibited PC-3 cell invasion compared with the control cells (Figure $2 \mathrm{~B}$; lane 1 vs. 2). Furthermore, ZA completely repressed cell invasion enhanced by the TGF- $\beta$ treatment, as showing that there is no significant difference between lane 2 and 4 in Figure 2B.

\section{Proteasome inhibitor overcomes ZA-induced down- regulation of NEDD9 expression}

The previous report evaluated that NEDD9 is ubiquitinated by AIP4, a specific E3 ligase, and subsequently degraded by $26 \mathrm{~S}$ proteasome [14]. As shown in Figure 3, a proteasome inhibitor MG132 clearly blocked the reduction of NEDD9 expression caused by the treatment with ZA. Based on this result, we speculated the possibility of ZA effect on the ubiquitination-proteasome system (UPS) targeted NEDD9 expression.

\section{ZA induces the ubiquitination of NEDD9}

In the immunoprecipitation assay using anti-NEDD9 antibody, the expression of poly-ubiquitinated NEDD9 was detected in PC-3 cells treated with ZA (Figure 4; lane 1 and 2). Moreover, addition with ZA markedly enhanced the poly-ubiquitination against NEDD9 under the block of degradation of NEDD9 using MG132 (Figure 4; lane 3 and 4).
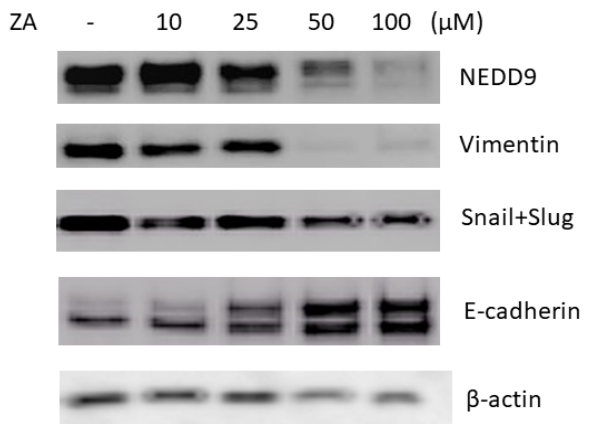

\begin{tabular}{|c|c|c|c|c|c|}
\hline \multirow{2}{*}{ Relativefold density } & \multicolumn{5}{|c|}{ ZA $(\mu \mathrm{M})$} \\
\cline { 2 - 6 } & 0 & 10 & 25 & 50 & 100 \\
\hline NEDD9 & 1 & 1.15 & 0.98 & 0.85 & 0.48 \\
\hline Vimentin & 1 & 0.93 & 1.04 & 0.79 & 0.73 \\
\hline Snail+Slug & 1 & 0.62 & 0.83 & 0.7 & 0.52 \\
\hline E-cadherin & 1 & 0.99 & 3.16 & 4.67 & 4.62 \\
\hline
\end{tabular}

Figure 1: Effects of zoledronic acid on expressions of NEDD9 and EMTassociated markers in PC-3 cells. PC- 3 cells were treated for $48 \mathrm{~h}$ with various concentrations of zoledronic acid as indicated. Cells treated with vehicle $(0.02 \%$ DMSO were used as the control. Treated cells were harvested, and total cell lysates were prepared and analyzed by western blot analysis using antibodies directed against the indicated proteins. To demonstrate equal loading amounts of total cell lysates, western blotting using anti- $\beta$-actin antibody was also performed (lowest panel).

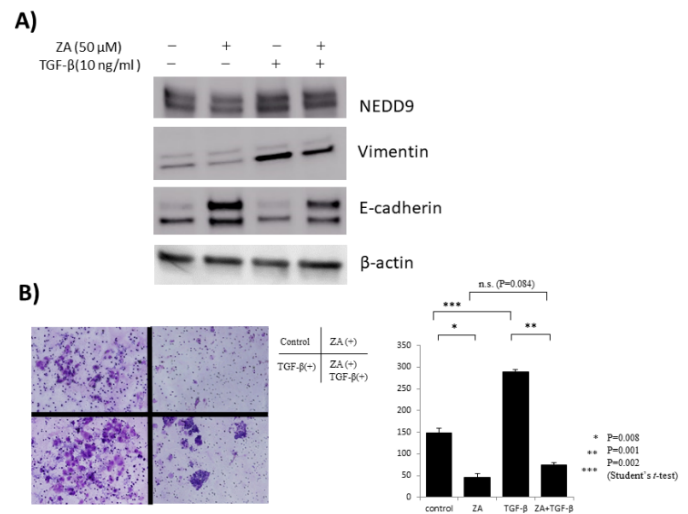

Figure 2: Suppressive effects of zoledronic acid on the EMT induction triggered by TGF- $\beta$ in PC-3 cells. PC-3 cells were incubated with TGF- $\beta(10 \mathrm{ng} / \mathrm{ml})$, ZA $(50 \mu \mathrm{M})$ or vehicle (control, $0.02 \%$ DMSO) for $48 \mathrm{~h}$. (A) Expression levels of NEDD9, epithelial and mesenchymal markers in PC-3 cells with/without treatment of TGF- $\beta$ and ZA were evaluated by western blotting. (B) Invasion assay in PC-3 cells. Three individual experiments were performed and mean \pm S.D. are presented; ${ }^{*} P,{ }^{* *} P,{ }^{* * *} P<0.05$ compared with the control (Student's t-test, $n=3$ ). N.S.; no significant difference.

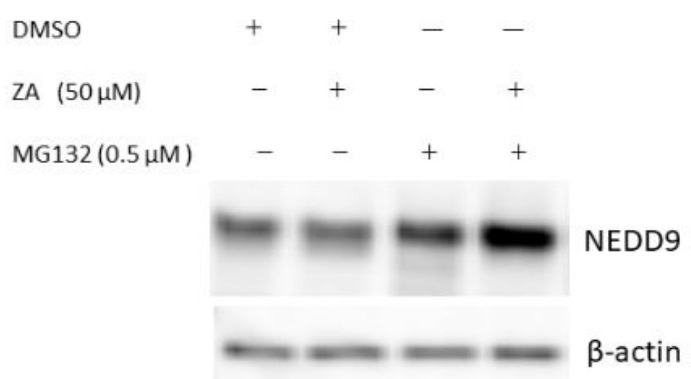

Figure 3: Effect of proteasome inhibitor on ZA-induced downregulation of NEDD9 expression. PC-3 cells were incubated with ZA $(50 \mu \mathrm{M})$, MG132 (0.5 $\mu \mathrm{M}$ ) or vehicle (control, $0.02 \%$ DMSO) for $48 \mathrm{~h}$. Expression levels of NEDD9 in PC-3 cells with/without treatment of ZA and MG132 were measured by western blotting.

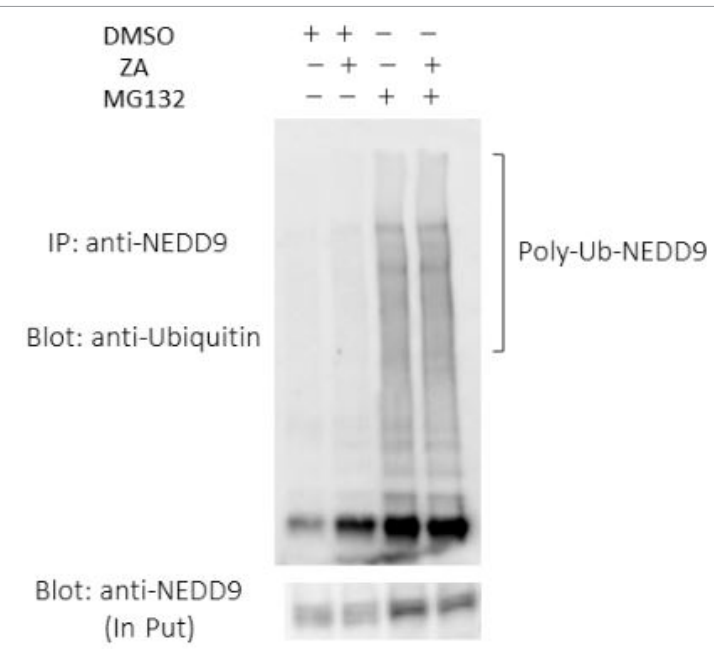

Figure 4: Enhancement of ubiquitination via exposure to $\mathrm{ZA}$ in $\mathrm{PC}-3$ cells. $\mathrm{PC}-3$ cells were incubated with ZA $(50 \mu \mathrm{M}), \mathrm{MG} 132(0.5 \mu \mathrm{M})$ or vehicle (control, $0.02 \%$ DMSO) for $48 \mathrm{~h}$. Expression levels of poly-ubiquitinated NEDD9 were detected using anti-ubiquitin antibody for the immunoprecipitated samples against antiNEDD9 antibody in PC-3 cells. 
Citation: Tanaka T, Morimoto K, Nakatani T (2018) Zoledronic Acid Suppresses Epithelial-to-Mesenchymal Transition and Invasion via Degradation of Ubiquitinated NEDD9 in PC-3 Prostate Cancer Cells. J Cancer Sci Ther 10: 080-084. doi: 10.4172/1948-5956.1000523

\section{Discussion and Conclusion}

Zoledronic acid (ZA), a third-generation bisphosphonate agent, has been shown to be a compound to treat prostate cancer bone metastasis and reduce skeletal-related events (SREs) in the clinical settings. Based on several basic researches, the bone-modifying agents (BMAs) including some bisphosphonates and a RANK-L (receptor activator of nuclear factor- $\kappa B$-ligand) inhibitor are thought to be a potent option for not only inhibiting the activation of osteoclasts but also preventing the tumor burden through the disturbance in the mitogen-based crosstalk among tumor, stromal and immune cells in the microenvironment of distant metastases $[11,15]$. In particular, our group reported that the nitrogen-containing bisphosphonates such as pamidronate and zoledronic acid exerted significant inhibition of bone cell- induced androgen receptor transcriptional activity in LNCaP prostate cancer cells [11]. On the other hand, the previous reports demonstrated the biological effects of ZA to reduce the EMT axis in some malignancies such as breast and prostate cancers $[16,17]$.

NEDD9 has been reported to be a key molecule that can regulate tumor aggressiveness, such as EMT, invasion, resistance to chemodrugs and dedifferentiation to stem cell via some down-stream cascades in cancer cells $[5,7,8,18]$. Regarding the turnover of NEDD9 expression, it was previously reported that NEDD9 is initially phosphorylated (targeted residue; serine 369) by a kinase sensitive to Hesperadin, followed by the $26 \mathrm{~S}$ proteasomal degradation of ubiquitinated NEDD 9 catalyzed by the specific ubiquitin E3 ligase, atrophin-1-interacting protein $4[14,19]$.

In this study, we focused on the biological action of ZA against the EMT and tumor invasion by regulating the NEDD9 expression in PC-3 prostate cancer cells. First, we initially elucidated that the treatment with ZA induced the reduction in the expressions of NEDD9 and its downstream EMT molecules (e.g., Snail/Slug). Simultaneously, ZA clearly inhibited tumor cell invasion triggered by TGF- $\beta$, a strong effector for EMT, in a boyden chamber assay. Additionally, we speculated the possibility that $\mathrm{ZA}$ affects the ubiquitin-proteasome system (UPS) pathway specific for regulating NEDD9 protein. As shown in Figure 3 and 4, we confirmed that the expression of NEDD9 is reduced by the treatment with $\mathrm{ZA}$ across the enhanced polyubiquitination and the subsequent degradation via $26 \mathrm{~S}$ proteasome in the PC-3 cells. To our knowledge, it is a first report to show the negative regulation of NEDD9 expression induced by the exposure to ZA.

Some clinical evidences that BMAs, such ZA and a RANKL inhibitor (i.e., denosumab), have strong effects on prolonged overall survival of CRPC patients with bone metastases and the period without the skeletal-related events (SREs) via marked inhibition of activity or formation of osteoclasts $[9,20]$. BMAs also have been reported to have anti-tumor effects on prostate cancer cells directly and indirectly in the basic experiments $[11,12,15]$, while there are few clinical evidence that the administration of these agents can exert the significant suppression of tumor burden in $\mathrm{PCa}$ patients. Furthermore, concerning the possibility of preventing bone metastasis of $\mathrm{PCa}$ via the administration of BMA, there are no beneficial data except for the significant delay in onset of bone metastases when denosumab was compared with placebo in CRPC patients without bone metastasis [21,22]. However, the conclusion cannot be drawn because those clinical trials have severe heterogeneity about the enrolled patients' background and the treatment schedules of some bisphosphonates and denosumab. From the results of this study, ZA is thought to have a high potential of inhibiting PCa cell invasion and metastasis, in concomitant with suppression of NEDD9 expression via the UPS-mediated degradation.
However, there is a limitation of no data regarding the effect of $\mathrm{ZA}$ on the cells with knockdown of NEDD9 expression. Our group previously demonstrated that knockdown of NEDD9 expression robustly induced the suppression of cell invasion [8]. Regrettably, we could not detect the biological effects on the cells under such a severe condition. In a future trial, the protective effect of ZA on bone metastasis may be clarified in high-risk M0 PCa patients, who are monitored by a modern imaging apparatus (e.g., 68Ga-PSMA/PET-CT, Whole body diffusion weighted MRI) with greater sensitivity to detect oligo bone metastases.

\section{Competing Interests}

The authors declare they have no conflict of interest.

\section{Authors' Contributions}

TT and KM conceived and designed the study. TT and KM performed the experiments. TT drafted the manuscript. TT, KM and TN assisted in performing data interpretation. TT and TN reviewed the manuscript for submission. All authors approved the final draft of the manuscript.

\section{Acknowledgements}

This research was financially supported in part by the Researcher Supporting Program of Osaka City University Graduate School of Medicine (KM and TT).

\section{References}

1. Tanaka T, Nakatani T (2011) New therapeutic strategies for castration-resistant prostate cancer. Recent Pat Anticancer Drug Discov 6: 373-383.

2. Thiery JP, Acloque H, Huang RY, Nieto MA (2009) Epithelial-mesenchymal transitions in development and disease. Cell 139: 871-890.

3. Thompson EW, Williams ED (2008) EMT and MET in carcinoma--clinica observations, regulatory pathways and new models. Clin Exp Metastasis 25: 591-592.

4. van der Pluijm G (2011) Epithelial plasticity, cancer stem cells and bone metastasis formation. Bone 48: 37-43.

5. Guerrero MS, Parsons JT, Bouton AH (2012) Cas and NEDD9 Contribute to tumor progression through dynamic regulation of the cytoskeleton. Genes Cancer 3: 371-381.

6. Kong C, Wang C, Wang L, Ma M, Niu C, et al. (2011) NEDD9 is a positive regulator of epithelial-mesenchymal transition and promotes invasion in aggressive breast cancer. PLoS One 6: e22666.

7. Izumchenko E, Singh MK, Plotnikova OV, Tikhmyanova N, Little JL, et al (2009) NEDD9 promotes oncogenic signaling in mammary tumor development. Cancer Res 69: 7198-7206.

8. Morimoto K, Tanaka T, Nitta Y, Ohnishi K, Kawashima H, et al. (2014) NEDD9 crucially regulates TGF-beta-triggered epithelial-mesenchymal transition and cell invasion in prostate cancer cells: involvement in cancer progressiveness. Prostate 74: 901-910.

9. Saad F, Eastham J (2010) Zoledronic acid improves clinical outcomes when administered before onset of bone pain in patients with prostate cancer Urology 76: 1175-1181

10. Saad F, Segal S, Eastham J (2014) Prostate-specific antigen kinetics and outcomes in patients with bone metastases from castration-resistant prostate cancer treated with or without zoledronic acid. Eur Urol 65: 146-153.

11. Tanaka T, Kawashima H, Ohnishi K, Matsumura K, Yoshimura R, et al. (2009) Indirect antitumor effects of bisphosphonates on prostatic LNCaP cells cocultured with bone cells. Anticancer Res 29: 1089-1094.

12. Hashimoto K, Masumori N, Tanaka T, Maeda T, Kobayashi K, et al. (2013) Zoledronic acid but not somatostatin analogs exerts anti-tumor effects in a model of murine prostatic neuroendocrine carcinoma of the development of castration-resistant prostate cancer. Prostate 73: 500-511.

13. Varol U, Degirmenci M, Karaca B, Atmaca H, Kisim A, et al. (2015) Zoledronic acid increases cytotoxicity by inducing apoptosis in hormone and docetaxelresistant prostate cancer cell lines. Tumour Biol 36: 779-786.

14. Feng L, Guedes S, Wang T (2004) Atrophin-1-interacting protein 4/human Itch is a ubiquitin E3 ligase for human enhancer of filamentation 1 in transforming growth factor-beta signaling pathways. J Biol Chem 279: 29681-29690. 
Citation: Tanaka T, Morimoto K, Nakatani T (2018) Zoledronic Acid Suppresses Epithelial-to-Mesenchymal Transition and Invasion via Degradation of Ubiquitinated NEDD9 in PC-3 Prostate Cancer Cells. J Cancer Sci Ther 10: 080-084. doi: 10.4172/1948-5956.1000523

15. Comito G, Pons Segura C, Taddei ML, Lanciotti M, Serni S, et al. (2017) Zoledronic acid impairs stromal reactivity by inhibiting M2-macrophages polarization and prostate cancer-associated fibroblasts. Oncotarget 8: 118-132.

16. Mani J, Vallo S, Barth K, Makarević J, Juengel E, et al. (2012) Zoledronic acid influences growth, migration and invasive activity of prostate cancer cells in vitro. Prostate Cancer Prostatic Dis 15: 250-255.

17. Schech AJ, Kazi AA, Gilani RA, Brodie AH (2013) Zoledronic acid reverses the epithelial-mesenchymal transition and inhibits self-renewal of breast cancer cells through inactivation of NF-kappaB. Mol Cancer Ther 12: 1356-1366.

18. Tikhmyanova N, Golemis EA (2011) NEDD9 and BCAR1 negatively regulate E-cadherin membrane localization and promote E-cadherin degradation. PLoS One 6: e22102.
19. Hivert V, Pierre J, Raingeaud J (2009) Phosphorylation of human enhancer of filamentation (HEF1) on serine 369 induces its proteasomal degradation. Biochem Pharmacol 78: 1017-1025.

20. Fizazi K, Carducci M, Smith M, Damião R, Brown J, et al. (2011) Denosumab versus zoledronic acid for treatment of bone metastases in men with castrationresistant prostate cancer: a randomised, double-blind study. Lancet 377: 813-822.

21. Hayes AR, Brungs D, Pavlakis N (2018) Osteoclast inhibitors to prevent bone metastases in men with high-risk, non-metastatic prostate cancer: A systematic review and meta-analysis. PLoS One 13: e0191455.

22. Smith MR, Saad F, Coleman R, Shore N, Fizazi K, et al. (2012) Denosumab and bone-metastasis-free survival in men with castration-resistant prostate cancer results of a phase 3, randomised, placebo-controlled trial. Lancet 379: 39-46. 